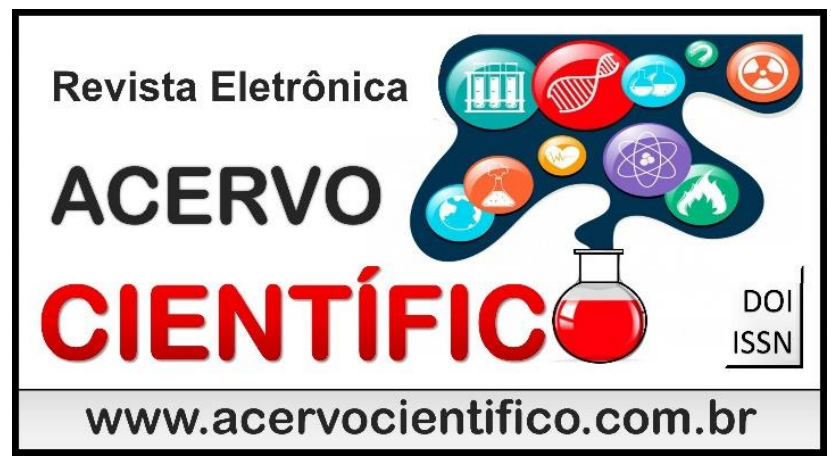

REVISÃO BIBLIOGRÁFICA

Recebido em: 1/2019

Aceito em: 2/2019

Publicado em: $3 / 2019$

\title{
O uso do QR Code em equipamentos médicos
}

\author{
The use of the QR Code in medical equipament \\ El uso del Código QR en equipos médicos
}

$\underline{\text { Valdecir de Godoy Borges }}^{1 *}$, Berthone Venâncio Soares ${ }^{2}$

\begin{abstract}
Resumo: Descrever como o uso do QR Code pode ser aplicado para realizar a calibração de equipamentos médicos, explicitando as inúmeras possibilidades que esta ferramenta possuiu. Trata-se de estudo com foco em pesquisa bibliográfica que faz a exposição do uso do QR Code como ferramenta para área médica e hospitalar, propiciando mostrar que este uso gera a união de requisitos de qualidade e de confiabilidade para um atendimento eficaz, na aplicação de controles em diversas áreas. A aplicação de softwares de leitura de QR Code permite com que se tenha a formação de códigos padrões que se destinam aos processos de controle em diversas áreas, e para os equipamentos médicos, o perfeito funcionamento e a calibragem são fundamentais, facilitando que se tenha o rastreamento do equipamento, a verificação do aparelho para a correta manutenção do mesmo, sendo esta preventiva ou mesmo de calibração diante de falhas, propiciando a redução do tempo de deslocamento de técnicos, em uso do QR Code em aplicativos de celulares ou tablet que direcionam os usuários em verificação automática para conferência das informações contidas no código. Conclui-se que a utilização do QR Code permite maior agilidade em calibração de equipamentos médicos.
\end{abstract}

Palavras-chave: QR Code, Smartphone, Engenharia Clínica.

\begin{abstract}
To describe how the use of the QR Code can be used to perform the calibration of medical equipment, explaining the countless possibilities that this tool possessed. This study with a focus on literature that makes the exposure from the use of the QR Code as a tool for medical and hospital care, providing show that this use generates the union of requirements of quality and reliability for an effective care, in the implementation of controls in several areas. The application of software to read QR Code enables you have the formation of codes patterns that are intended to control processes in various areas, and for medical equipment, the perfect functioning and calibration are fundamental, easing that has the tracking of equipment, checking the clip for proper maintenance of the same, being this maintenance or calibration even in the face of failures, leading to reduction in the time of displacement of technicians, in use of the QR Code in applications for mobile phones or tablets that directs users to automatically check for conference of the information contained in the code. The study concluded that the use of the QR Code enables greater agility in calibration of medical equipment.
\end{abstract}

Keywords: QR Code, Smartphone, Clinical Engineering.

1 Especialista em engenharia clinica pelo hospital Albert Einstein, São Paulo/SP.

*E-mail: marxboetila@gmail.com

${ }^{2}$ Coordenador de Engenharia Clínica, Diretoria de Engenharia e Manutenção Hospital Albert Einstein, São Paulo /SP. 
Resumen: Describir como el uso del Código QR puede ser aplicado para realizar la calibración de equipos médicos, exploniendo las inúmeras posibilidades que esta herramienta posee. Se trata de estudio com atención en pesquisa bibliográfica que hace la exposición del uso del código QR como herramienta para área médica y hospitalar, posibilitando demuestrar que este uso genera la unión de requisitos de calidad y de confianza para una atención eficaz, en el aplicación de controles en distintas áreas. Resultados: El aplicação de softwares de lectura de código QR permite que se haga la formación de códigos padrones que se destinan a los procesos de contról en distintas áreas, y para los equipos médicos, la perfecta acción y la calibración son fundamentales, facilitando que se tenga el rastreo del equipo, la verificación del aparato para el correcto mantenimiento de este, siendo esta preventiva o mismo de calibración ante fallas, propiciando la reducción del tiempo de deslocamento de técnicos, en uso del código QR Code en aplicativos de teléfonos o tablet que direccionan los usuarios en verificación automática para conferir las informaciones contenidas en el código. Se Concluye que la utilización del código QR permite mas agilidad velocidad en calibración de equipos médicos.

Palabras clave: QR Code, Smartphone, Ingeniería Clínica.

\section{INTRODUÇÃO}

O uso do QR Code na atualidade se transformou em uma ferramenta relevante e, na área hospitalar apresenta inúmeras utilidades, tendo em vista que as informações que este contém podem ser facilmente lidas por meio de celulares ou outros dispositivos em aplicação de softwares de leitura.

As instituições hospitalares precisam, constantemente, do cuidado com equipamentos e a Gseis Engenharia propicia um serviço de engenharia clínica com qualidade nos serviços de saúde, que dependem de tecnologias médicas com alta qualidade e confiabilidade. Portanto, o uso do QR Code visa garantir a qualidade nos serviços prestados em aplicação de novas tecnologias de calibração.

A atuação por meio da aplicação do QR Code torna a atividade de engenharia clínica mais flexível com foco em facilitar o trabalho da equipe da instituição, demonstrando assim maior transparência, melhoria contínua, sustentabilidade e compromisso com o cliente para que realize a correta calibração dos equipamentos médicos.

O trabalho expressa a perspectiva de uso do QR Code, partindo da exposição de suas principais utilizações dentro da engenharia clínica, aplicando na parte teórica informações bibliográficas acerca do tema para, posteriormente, expor como ocorre o uso do QR Code por meio de aparelho celular, demonstrando tal uso para área de equipamentos médicos.

Portanto, este trabalho busca relatar a aplicação do uso do QR Code por meio de empresa, cujo foco está em realizar a calibração de equipamentos médicos, utilizando aparelhos celulares em aplicação de softwares de leitura do código, que pode se apresentar como solução moderna para aplicação na área hospitalar.

\section{RESULTADOS E DISCUSSÃO}

Atualmente, o QR Code que inicialmente foi desenvolvido para rastrear veículos passou a ser o mais famoso código de barras e, na área hospitalar surge como uma ferramenta que possui inúmeras utilidades. As informações que um QR Code armazena podem ser lidas por meio de um celular ou outros aparelhos eletrônicos.

Por entender que a aquisição dos instrumentos de calibração, muitas vezes, envolve um alto custo para as unidades, a Gseis Engenharia disponibiliza ao mercado os serviços de locação de padrão/simulador sendo este seu principal diferencial. Oferecendo soluções mais modernas e sempre com a garantia do melhor padrão/simulador do mercado, tudo conforme as exigências da Norma, a Gseis tem como um dos seus principais objetivos trabalhar com a transparência e honestidade com os clientes, fazendo com que estes tenham total confiabilidade nos serviços prestados. 
A Gseis Engenharia tem o foco em sua missão, que é trabalhar oferecendo qualidade aos serviços, gerando confiabilidade no mercado, e tendo a visão de ser referência nos serviços de calibração em equipamentos médicos.

O diferencial, que é proposto pela Gseis Engenharia, é a locação de padrões calibrados para hospitais que possuem o serviço de engenharia clínica, fazendo com que a qualidade nos serviços de saúde, que depende das tecnologias médicas, seja de alta qualidade e confiabilidade propiciando manter a melhoria contínua.

Dentro de uma área que é bem restrita, a engenharia para calibração de equipamentos médicos, a utilização do QR Code surge a fim de facilitar o trabalho da equipe demonstrando, com isso, maior transparência, melhoria contínua, sustentabilidade e compromisso com o cliente.

A identificação de equipamentos por meio de um controle patrimonial, geralmente, é realizada aplicando um código numérico e esta situação pode comprometer o correto controle. Assim, o uso de recursos tecnológicos, bem como computacionais tem como objetivo facilitar o registro para propiciar um melhor controle gerencial.

Dessa forma, a aplicação de um sistema de codificação propicia com que se tenha maior facilidade para controle de quantidade de equipamentos existentes em um hospital, sendo registrados ainda aspectos como valor do mesmo, a data de aquisição e local em que este está instalado ou alocado, sendo informado também qual o estado de funcionamento. Todas essas informações podem ser identificadas por meio do sistema de codificação que permite a leitura por intermédio de uso do celular, o que se apresenta como uma forma mais simples para os registros.

Garantir a segurança e eficácia nos processos de calibração de equipamentos médicos se apresenta como aspecto relevante para o suporte da área médica e hospitalar, e o trabalho de calibração realizado por meio do uso do QR Code surge como um facilitador, tendo em vista que este pode simplificar o trabalho de técnicos, de engenheiros, de enfermeiros e de médicos. Da mesma forma se busca mostrar que esta ferramenta pode reduzir o armazenamento e a impressão de documentos, bem como reduzir o tempo de deslocamento de técnicos, propiciando facilitar o controle de inventário de equipamentos de forma remota.

Em uma perspectiva tecnológica, O QR Code foi desenvolvido pela empresa Denso Wave, subsidiária da Toyota, segundo exposição de Gabriel (2010), com foco em rastrear os veículos durante a produção e, em seguida se transformou em um famoso código de barras existente, chamando a atenção de profissionais de todas as áreas, que perceberam as inúmeras potencialidades estratégicas que estes códigos oferecem.

$\mathrm{Na}$ área hospitalar ainda é uma ferramenta que tem inúmeras possibilidades como, por exemplo, no controle de engenharia, no controle de medicamentos, na identificação de pacientes, na identificação medicamentosa e outras. Quando surgiu, o código era usado na indústria, para organizar melhor o estoque. Atualmente, já é comum encontrar em revistas, em anúncios, em cartazes e até embalagem de produtos.

A informação contida em um QR Code pode ser facilmente lida por meio de um leitor QR instalado em um tablet ou celular. Os softwares de leitura são facilmente encontrados em vários sites especializados e há um grande número de softwares fornecidos gratuitamente. A formação de códigos padrões tem sido fundamental para facilitar e ajudar em inúmeros processos de controle em diversas áreas.

Segundo Okada e Souza (2011), o QR Code tem um amplo aspecto de utilização, pois os usuários são direcionados para um link direto para sites, livros ou redes sociais, aplicando o acesso propiciando por meio do uso do celular em conexão com internet.

A denominação QR Code recebe sua designação do inglês, cujo significado é "resposta rápida", sendo um código que pode ser escaneado pela maioria dos aparelhos celulares que contém uma câmera, um software adequado e internet para a leitura do mesmo. O principal objetivo do uso desta ferramenta está na praticidade em transmitir informações codificadas em alta velocidade, mesmo com imagens de baixa resolução (OKADA; SOUZA, 2011). 
Segundo Gabriel (2010), para ser possível digitalizar um QR Code é necessário que o dispositivo móvel possua, além de câmera fotográfica, um leitor de mobile tagging apropriado para o sistema operacional do aparelho.

A norma ISO 18004/2006 define o QR Code como:

"um grafismo matricial constituído por um conjunto de módulos nominalmente quadrados dispostos em um padrão global na forma de quadrado, que inclui um padrão único, com localizador encontrado nos três cantos do símbolo e destinado a facilitar a localização de sua posição, tamanho e inclinação" (www.iso.org).

A forma como as informações são exibidas, por meio do QR Code, também denominado de Quick Response Code, pode ocorrer em registros horizontais ou verticais, sendo este um aspecto considerado bidimensional, e tal característica é o que propicia que os códigos QR sejam capazes de armazenar uma grande quantidade de dados, bem maior do que os códigos de barras.

Segundo Soon (2008), o QR Code é um código do tipo matricial com uma estrutura de células dispostas em um quadrado, e consiste em padrões de funcionalidade para fazer a leitura de maneira fácil da área de dados, na qual esses são armazenados. Estas células são módulos pretos e brancos que contém informação binária, sendo o módulo branco correspondente ao ' 0 ' e o preto correspondente ao ' 1 '.

O que faz um QR Code ter muito mais vantagens do que um código de barras convencional é que este contém informações em ambas as direções, tanto na vertical quanto na horizontal, enquanto um código de barras convencional contém informações somente na horizontal. Desse modo, um QR Code pode conter uma quantidade de dados consideravelmente maior do que um código de barras comum.

Assim, dentro da área de saúde em que o professional precisa, muitas vezes, do acesso rápido aos registros de equipamentos e estes precisam estar devidamente calibrados, o acompanhamento deste enfoque é primordial para que não ocorram problemas em atendimento.

No código de barras linear ou unidimensional, a quantidade de dados depende do comprimento e não da altura, visto que os dados são armazenados nas barras, enquanto no QR Code, os dados são dispostos em duas dimensões, na horizontal e na vertical, possibilitando um melhor aproveitamento do código.

Nesse sentido, a capacidade de armazenamento de dados que se alcança por meio do uso do QR Code é bem maior que o que se aplica por meio do tradicional código de barras, tendo em vista que um QR Code aplica caracteres alfabéticos, bem como números e símbolos binários relacionados com o alfabeto Japonês como o Kanji e Kana, tendo assim a capacidade de combinação de diversos símbolos combinados em até dezesseis símbolos ou um símbolo grande que pode alcançar até 7.089 caracteres em oposição ao código de barras, que apenas comporta o máximo de vinte dígitos.

Ao lado desta capacidade de armazenamento de dados que o QR Code apresenta se tem a vantagem de que esse pode ser digitalizado em aplicação de diversos ângulos, ou seja, pode ser aplicado em 360 graus, permitindo inclusive codificar os dados usando um espaço bem menor do que o que se aplica ao código de barras tradicional.

De acordo com Soon (2008), um QR Code é composto, primordialmente, de Finder Pattern, Alignment pattern, o Timing pattern e da Quiet zone, sendo o Finder Pattern identificado pelos três quadrados grandes nos cantos, sendo este um diferencial em relação à qualquer outro código bidimensional. Assim, possibilitam a leitura do código em qualquer ângulo. Segundo Soon (2008), estes marcadores de orientação detectam a posição, o tamanho e o ângulo do código a ser lido.

Os timing patterns são as linhas pontilhadas que ligam os Finder Patterns tanto na horizontal quanto na vertical. Segundo Soon (2008), os timing patterns são padrões para a identificação da coordenada central de cada módulo no QR Code, sendo possível a correção do código quando há distorção ou erro na disposição dos módulos. Já os Alignment pattern permitem a correção de distorções locais. 
Os Quiet Zone são as margens de folga presentes nos quatro lados do QR Code, e estas auxiliam no reconhecimento rápido e preciso da imagem.

Format Information indica a versão do código, o nível de correção de erro. Um mesmo código pode conter áreas de dados de outros códigos, nos quais os mesmos dados podem ser obtidos tanto a partir do QR Code superior, quanto ao se juntar a informação dos quatro códigos inferiors e o separator information separa as informações contidas no código QR.

A data área é o espaço em que, efetivamente, os dados originais são dispostos de maneira codificada em números binários, incluem também dados que têm funcionalidade de correção de erros, Soon (2008) afirma que a informação é codificada do tipo de dados original para números binórios, que serão convertidos em módulos brancos e pretos quando dispostos.

O controle de equipamentos médicos hospitalares surge como uma maneira de verificação e de controle de quais equipamentos existem na instituição, bem como em identificar quais desses estão sob cuidados de empresa que seja prestadora de serviços, de maneira que o registro em lista e o controle de informações consideradas como básicas de cada equipamento nem sempre propicia uma administração eficiente para os mesmos.

Nesse sentido, as instituições hospitalares necessitam de um bom gerenciamento de todos os equipamentos para que o atendimento médico hospitalar seja percebido como eficiente, bem como este gerenciamento sirva para facilitar nos processos de certificações de instituições.

A correta identificação de equipamentos, por meio da engenharia clínica, permite a correta rastreabilidade dos mesmos em acompanhamento de número de patrimônio da mesma forma que em registro de codificação.

De forma geral, o controle patrimonial implica o uso de código numérico, e esse nem sempre serve corretamente para a devida identificação do equipamento e pode acabar comprometendo o eficiente controle patrimonial.

Portanto, é importante que o registro seja feito de forma a ser incorporado aos recursos tecnológicos e computacionais e, assim, o uso do QR Code em aplicação de leitura por meio do celular facilita 0 acompanhamento de informações do equipamento, praticamente, em tempo real.

A aplicação de um sistema de codificação tem como interesse propiciar com que o gestor tenha a correta identificação dos equipamentos, bem como as quantidades existentes, monitorando também o valor, a data de compra e o local em que estes equipamentos estão alocados dentro da instituição hospitalar, além de possibilitar o gerenciamento do estado de funcionamento de cada um. Assim, essas informações ao serem disponibilizadas por meio de um sistema de codificação permite uma gestão direcionada para o acompanhamento da correta manutenção e funcionalidade de cada um, permitindo que a codificação em aplicação de registro por meio do QR Code facilite o acesso para o banco de dados, gerando assim um controle mais eficiente em informações agrupadas.

Com estes dados se facilita o envio de informações específicas ao vincular o equipamento a um sistema de gerenciamento ideal em nuvem, sendo tanto para cadastro de novos equipamentos, quanto para verificação de status do mesmo, nas rotinas dos técnicos, bem como em rondas, sendo muito fácil visualizar os dados em tempo real, tendo em mãos um celular ou tablet com software de leitura QR Code.

Também para fracionar os setores no direcionamento específico no caso de uma gerente de enfermagem de UTI, por exemplo, uma vez que esta apenas poderá visualizar os equipamentos do seu setor e não de outros, o que inibe também a migração de equipamentos de um setor a outro, no caso do usuário, ele não tem liberdade de atuação diretamente no equipamento, podendo apenas pedir chamados de serviços e acompanhar os mesmos, inclusive, dando notas ao serviço desenvolvido.

Algumas alterações como calibração, testes de segurança elétrica preventiva saem automaticamente para o setor interessado com foto do equipamento e outros dados relevantes para registro de equipamento e o gerenciamento e a rastreabilidade do mesmo dentro do hospital, fazendo com que o processo seja mais 
simples e confiável e deixando ao técnico mais tempo para se dedicar, com mais qualidade, aos problemas da equipe de engenharia hospitalar.

Para ilustrar como funciona o registro do QR Code, abaixo segue a imagem de um código, no qual está escrito: Turma de pós-graduação em engenharia clínica 2016/2107.

Figura 1 - QR Code Turma de Engenharia Clinica 2016/2017

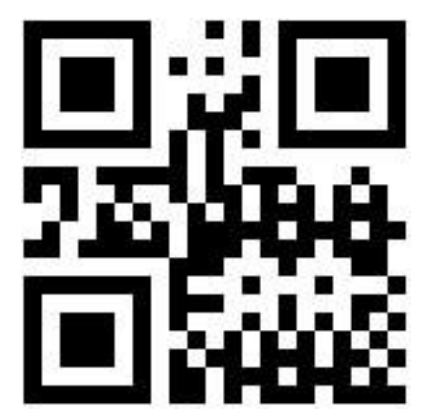

Fonte: o autor

Inicialmente, em volta de todo código há três marcas maiores de referência para que o leitor saiba onde inicia e onde termina a leitura, sendo três os pontos de referência mostrados na figura, que ficam mais evidentes no QR Code e abaixo não existe nenhuma informação. Nas partes brancas do QR Code surgem informações basicamente divididas em 8 bytes.

Nos três quadrados maiores, mas alguns QR Code podem ter dois ou apenas um, ao lado dos quadrados superiores se encontra a versão. Existem atualmente aproximadamente 40 versões que podem ir de $21 \times 21$ até $177 \times 177$.

Cada formação de intervalos entre cores pretas e brancas constrói padrões ou espaços e formas de figuras, que compõem as palavras ou caracteres. Quanto maior o número de caracteres, dependendo do formato que estiver usando as formas, em preto e branco, a informação exata que está contida no sistema de códigos QR Code será menor.

Assim, a aplicação desta ferramenta em diversas atividades na área hospitalar é relevante por agilizar as informações ao ser possível que celulares sejam aplicados para simplificar o trabalho dos profissionais em processamento de informações.

\section{CONSIDERAÇÕES FINAIS}

Ao longo do texto se procurou demonstrar que o uso do QR Code, por empresas de engenharia clínica, evidenciam os aspectos positivos para o uso desta ferramenta, uma vez que se pode dizer que esta ferramenta pode ter muita utilidade, permitindo expor que o uso do QR Code simplifica o trabalho de técnicos, de engenheiros, de enfermeiros e de médicos, minimizando as condições de erro, fornecendo uma maior segurança quanto ao armazenamento e ao processamento de informações. Da mesma maneira, o uso do QR Code reduz a documentação, uma vez que as informações ficam armazenadas no site que gerencia os dados, propiciando redução no tempo de acesso aos mesmos e, ainda, pode-se concluir que o uso desta ferramenta propicia controle sobre o patrimônio, ou seja, os equipamentos médicos e hospitalares, gerando também segurança para os pacientes que são atendidos em aplicação dos mesmos, por estarem continuamente sendo monitorados para que em identificação de falhas, estas possam ser resolvidas com a maior brevidade 


\section{REFERÊNCIAS}

1. ATOMURL. O que é código QR? Disponível em: http://atomurl.net/qrcode/. Acesso em: 26 ago. 2017.

2. BAJUR T. Controle de inventário, saiba o que você está fazendo errado. 2016. Disponível em: https://blog.arkmeds.com/2016/08/29/controle-de-inventario-saiba-o-que-fazer/ . Acesso em: 28 ago. 2017.

3. BAMPI JUNIOR D. Estudo sobre a técnica de códigos de barras bidimensional - QR Code. Eventos. 2014. Disponível em: http://eventos.ifc.edu.br/wp-content/uploads/sites/5/2014/08/ESTUDO-SOBRE-A-TECNICA-DECODIGOS-DE-BARRAS-BIDIMENSIONAL-QR-CODE.pdf. Acesso em: 26 ago. 2017.

4. BERNARDO L. Aprenda a usar o QR Code: código 2D permite rápido acesso a links e outras informações. 2016. Disponível em https://vejasp.abril.com.br/cidades/como-usar-qrcode/ . Acesso em: 01 set. 2017.

5. BOYLESTAD RL. Introdução a análise de circuitos. 12. ed. São Paulo: Pearson, 2004. 591.p.

6. COPETTI C, GHISLENI TS. Mobile marketing: a tecnologia QR Code utilizada em ação da Heineken. Disciplinarum Scientia - Sociais Aplicadas, 2012; 8(1):59-69.

7. DENSO WAVE. About 2D Code. Disponível em: http://www.denso-wave.com/qrcode/aboutqr-e.html . Acesso em: 28 ago. 2017.

8. GABRIEL M. Marketing na era digital. São Paulo: Novatec, 2010. 424 p.

9. HAYKIN S. Sistemas de comunicação: analógicos e digitais. 4. ed. São Paulo: Artmed, 2007. 1270 p.

10. ISO. ISO/IEC 18004:2015(en). Information technology — Automatic identification and data capture techniques — QR Code bar code symbology specification. 2015. Disponível em: http:/www.iso.org/obp/ui/\#iso:std:isoiec:18004:ed3:v1. Acesso em: 27 ago. 2017.

11. OKADA SI, SOUZA EMS. Estratégias de marketing digital na era da busca. Revista Brasileira de Marketing, $2011 ; 10(1): 46-72$.

12. SESC SÃO PAULO. Qual a história por trás de um QR Code? 2013. Disponível em: https://www.sescsp.org.br/online/artigo/7196_QUAL+A+HISTORIA+POR+TRAS+DE+UM+QR+CODE . Acesso em: 25 ago. 2017.

13. SILVA O. Leitor QR Code. Microsoft. Disponível em: https://www.microsoft.com/pt-br/store/p/leitorqrcode/9wzdncrfjon4. Acesso em: 01 set. 2017.

14. SOON TJ. QR Code. Synthesis Journal. Section three. iTSC Information Thechnology Standard Comitee. Singapore, 2008, p. 59-78.

15. XAVIER F. QR Code: entenda o que é e como funciona o código. 2011. Disponível em: https://www.techtudo.com.br/dicas-e-tutoriais/noticia/2011/03/um-pequeno-guia-sobre-o-qr-code-uso-efuncionamento.html. Acesso em: 05 set. 2017.

16. YOUNG H, FREEDMAN R. Física III: eletromagnetismo. 12. ed. Saraiva, 2009. 352 p. 